\title{
VIEWPOINT
}

\section{Permissive hypofiltration}

Lakhmir S Chawla*1,2, John A Kellum ${ }^{3,4}$ and Claudio Ronco 5

\begin{abstract}
Acute kidney injury (AKI) is a syndrome with a multitude of causes and is associated with high mortality and a permanent loss of renal function. Our current understanding of the most common causes of AKI is limited, and thus a silver bullet therapy remains elusive. A change in the approach to AKI that shifts away from the primary composite endpoint of death/ dialysis, and instead focuses on improving survival and mitigating permanent renal damage, is likely to be more fruitful. We suggest that the current approach of augmenting renal function by increasing the renal blood flow or glomerular filtration rate during AKI may actually worsen outcomes. Analogous to the approach towards adult respiratory distress syndrome that limits ventilator-induced lung injury, we propose the concept of permissive hypofiltration. The primary goals of this approach are: resting the kidney by providing early renal replacement therapy, avoiding the potentially injurious adverse events that occur during AKI (for example, fluid overload, hypophosphatemia, hypothermia, and so forth), and initiating therapies focused on improving survival and mitigating permanent loss of kidney function.
\end{abstract}

\section{Introduction}

Acute kidney injury (AKI) is a disorder that often complicates the hospital course of patients and is associated with a significant mortality risk. Those patients with the most severe forms of AKI, who develop such significant solute or fluid imbalance that they are usually treated with renal replacement therapy (RRT), experience a hospital mortality of approximately $60 \%$ [1]. In spite of improvements in care, the mortality of AKI remains unacceptably high [2].

*Correspondence: minkchawla@gmail.com

'Department of Anesthesiology and Critical Care Medicine, George Washington University Medical Center, 900 23 $3^{\text {rd }}$ Street, NW, RILF, Room G-105, Washington, DC 20037, USA

Full list of author information is available at the end of the article
AKI is a clinical syndrome that is associated with the acute loss of kidney function, as evidenced by a drop in the glomerular filtration rate (GFR). The most common causes of AKI in developed countries are sepsis, circulatory shock, major cardiovascular surgery, and heart and liver failure [1]. Because decreased renal blood flow (RBF) is usually assumed to play a role in each of these conditions, the traditional teaching approach for AKI is that restoration of RBF is critical. The focus of therapeutic intervention in AKI for the past 40 years has thus been to restore RBF and to minimize nephrotoxic exposure. Therapeutic clinical trials of AKI have focused on increasing the RBF and GFR by utilizing a variety of drugs (for example, dopamine, anaritide, fenoldopam) [3]. Unfortunately, this approach has yielded no successful therapy for the treatment of AKI.

\section{Our understanding of the pathophysiology of AKI is insufficient}

AKI is a clinical syndrome with a multitude of causes. Like any syndrome, AKI can be separated into susceptibility and exposure. The most common susceptibilities for AKI are advanced age and chronic kidney disease (CKD). The most common exposure related to AKI is sepsis, which is associated with 45 to $50 \%$ of all cases of AKI [4]. Of note, available preclinical and human data suggest that septic-associated AKI is not associated with tubular necrosis $[5,6]$. These same studies demonstrate that $\mathrm{RBF}$ in sepsis is typically increased, and that, despite this augmented RBF, septic patients still experience a drop in GFR and develop AKI (reviewed in [6]). In addition, the few studies that have assessed histopathology in septic patients do not show evidence of tubular necrosis [7]. The failure to develop appropriate therapeutic agents for AKI may thus be due to the fact that the therapeutic agents being considered work via increasing the GFR by increasing RBF (for example, anaritide) - which is unlikely to benefit those patients with sepsis, the most common exposure associated with AKI.

It is logical to try and assist a failing organ by treating the underlying cause. Once an organ is injured, however, trying to force that organ to work harder may not be the best approach. In fact, this approach has been shown to be counter-productive in other areas of critical care. For 
example, the focus of therapy during myocardial infarction is twofold: treat the underlying cause and rest the injured organ. For example, when patients sustain a significant myocardial infarction, if ventricular function is inadequate after treatment (for example, angioplasty) then it is common to place an intra-aortic balloon pump (or in extreme cases a ventricular assist device) to decrease the workload on the heart [8]. Similarly, the therapeutic intervention in adult respiratory distress syndrome (ARDS) is to decrease the tidal volume, sometimes even to the point of allowing respiratory acidosis (permissive hypercapnia), in order to avoid exacerbating lung injury and thereby to improve outcomes [9].

\section{Lessons from ARDS and potential for harm}

AKI and ARDS are syndromes that have much in common. Both have multiple etiologies; and the most common cause for both ARDS and AKI is sepsis. Both syndromes are associated with increased mortality; and both affect critically ill patients, contributing to an increased risk of death. In addition, both syndromes typically involve a mechanical intervention for organ support, RRT and mechanical ventilation, respectively. However, the clinical trial endpoints of focus are quite different for AKI and ARDS. In ARDS, the endpoints utilized in clinical trials are most often both short-term disability (30-day mortality, ventilator-free days) and long-term disability. For AKI, however, the endpoints are typically only short term: 30-day mortality and need for RRT. The need for RRT is akin to the need for intubation and mechanical ventilation in ARDS and yet the two endpoints are treated very differently. This difference is critical. The notion that a drug or intervention can prevent the need for mechanical ventilation in a patient with severe ARDS is not realistic. ARDS causes rapid deterioration in pulmonary function and organ support (for example, mechanical ventilation) is instituted early. Because the endpoint in AKI is need for RRT, there is an implicit expectation that a drug or intervention can alter the clinical trajectory of severe AKI within a few days and furthermore that RRT can be safely delayed awaiting renal recovery. With the advent of more biologically compatible membranes and physiologic RRT fluids, there has been a secular trend of initiating RRT sooner. Therefore, even if diagnostic techniques improve our time to diagnosis of AKI, do we expect a therapeutic drug or intervention to forestall the need for organ support within a short time frame (that is, 2 to 3 days)? Unlike opening an artery for the treatment of myocardial infarction, the kidney's response to injury is more complex; the renal vasculature, glomeruli, renal tubules, and interstitium do not respond monolithically. To expect an intervention or drug to heal the kidney and all its complex components in a few days is not biologically plausible.
One of the lessons of ARDS is thus to focus on realistic endpoints. Instead of short-term mortality and need for RRT, we propose that the more appropriate short-term endpoints in studying AKI should be short-term mortality and RRT-free days, or even AKI-free days (similar to the endpoints in ARDS).

Another critical lesson from ARDS is the potential to do harm. The mainstay of treatment for ARDS is to avoid ventilator-induced lung injury [9]. A key advance in the treatment of ARDS was the recognition that mechanical ventilation performed incorrectly can be injurious [9]. Similarly, the way that patients with AKI are treated can also be injurious. RRT must be prescribed in a thoughtful fashion to avoid significant adverse events. For instance, the use of intermittent hemodialysis in critically ill patients can result in significant hypotension and persistent intestinal ischemia $[10,11]$.

\section{Fluid overload in acute kidney injury}

Because classic teaching states that hypovolemia is the most common reversible cause of AKI, the standard treatment for AKI is a trial of volume (for example, crystalloid infusion). For the vast majority of clinicians, the standard approach to AKI once it is recognized is as follows: rule out reversible causes of AKI, discontinue diuretics, administer a trial of volume, stop any nephrotoxic drugs, and rule out obstructive nephropathy. Once the patient is deemed optimally resuscitated, a trial of loop diuretics is usually conducted. This approach is reasonable and represents the recommended approach [12]. However, this approach also offers the opportunity to do harm.

A trial of volume administration is a core feature of most empiric AKI treatment. Not surprisingly, fluid overload is common in patients with AKI [13]. Fluid overload has been shown to be associated with increased mortality and increased length of stay in multiple studies, and has an independent effect on mortality even after the severity of illness and the degree of AKI are taken into account [13-15]. This association has been shown in adults and children, and prospective trials have shown that interventions which reduce fluid overload lead to more rapid liberation from mechanical ventilation and less renal failure $[16,17]$.

\section{Renal replacement therapy in acute kidney injury}

For those patients with AKI who require RRT, the technical aspects of RRT have become increasingly important. Contemporary RRT is associated with higher dosing of RRT as compared with historical controls. When patients are subjected to higher doses of RRT, they experience improved solute clearance and faster resolution of electrolyte and acid-base imbalances. However, these patients may have a higher incidence of inadequate plasma antibiotic levels, hypophosphatemia and hypothermia. 
Pharmacology studies assessing antibiotic levels in patients undergoing continuous renal replacement therapy (CRRT) with contemporary dosing found that antibiotic levels were inadequate for up to $75 \%$ of the antibiotics tested [18]. Appropriate and adequate antibiotics has been a cornerstone of sepsis therapy $[19,20]$, and studies such as these endorse the notion that antibiotics can be rendered less useful if the CRRT and the drug dosing are not harmonized. Similarly, patients undergoing CRRT are susceptible to hypophosphatemia [21]. Hypophosphatemia can have multiple negative consequences including, but not limited to, hemolysis, respiratory failure, rhabdomyolysis, delirium and neutrophil dysfunction [22,23]. Another potential complication of RRT is hypothermia. Because of the extracorporeal circuit, inadequate warming of replacement fluid/dialysate can lead to hypothermia [24]. In a large multicenter study of more than 10,000 patients, hypothermia has been shown to be a major risk factor associated with increased risk of mortality in critically ill patients [25]. Large well-powered clinical trials of increased doses of RRT did not protocolize these aspects of RRT $[21,26]$, and some investigators have suggested that inadequate control of these parameters may have had a significant negative impact.

Early institution of RRT is likely to have multiple positive effects in AKI, including improved fluid balance and the capacity to rest the injured kidney. However, the manner in which RRT is conducted in the ICU appears to be important. RRT must be conducted with the appropriate management of drug dosing, thermal control, volume and electrolyte balance.

\section{Interplay of acute kidney injury and chronic kidney disease}

Classic teaching regarding patients who survive an episode of AKI - in particular, acute tubular necrosis states that these patients achieve full or nearly full recovery. This notion was based on multiple studies that followed survivors of AKI after hospital discharge [27]. More recently, from 2008 through 2012, multiple studies assessing unique cohorts of patients demonstrated that patients who survive an episode of AKI have a significant risk for progression to advanced CKD (stage 4/5) [28-33]. Moreover, there are multiple studies that have linked severity of AKI with increased risk of CKD progression $[29,30,34]$. In short, patients who experience a severe episode of AKI are at risk for incident CKD, and those patients with pre-existing CKD are at risk for accelerated progression of CKD.

\section{Choice of endpoints for clinical trials in AKI}

As discussed earlier, the short-term composite endpoint of death/RRT may not be appropriate. Because subsets of
AKI patients tend to progress to CKD, medium-term outcomes in AKI should also be assessed. Similar to the pathophysiology of ARDS, wherein a fibroproliferative phase follows the initial exudative phase, subsets of patients with AKI experience ongoing fibrosis that tends to persist after injury. Clinical epidemiologic studies confirm that about 15 to $20 \%$ of patients who experience AKI rapidly progress to advanced-stage CKD within 20 to 24 months [28]. Given that CKD is a significant risk factor for cardiovascular events, patients who survive AKI and progress to CKD are more likely to experience cardiovascular events [35].

In aggregate, we propose that endpoints for clinical trials of AKI should be short term and medium term. The primary short-term endpoints should be 30-day mortality and AKI-free days. Medium-term endpoints should include 90-day to 180-day outcomes to determine whether permanent loss of renal function and associated cardiovascular events can be mitigated. The composite endpoint of major adverse kidney events has been proposed to include each of these end points.

\section{Hypothesis: permissive hypofiltration}

The approach we propose is based on four broad themes: (1) focus on the underlying process causing AKI, since there are many different mechanisms that cause AKI; (2) when a patient is experiencing AKI, augmenting the GFR and RBF may not be helpful and may in fact be harmful; (3) current and recommended interventions for AKI have the capacity to do harm; and (4) interventions should be focused on durable renal recovery and improving survival.

The first theme is obvious of course, but has important consequences when enacted. This theme recognizes that septic-associated AKI is different from nephrotoxic AKI, which is different from ischemic AKI caused by profound global hypotension (for example, cardiac arrest). The treatment for all AKI is therefore not necessarily about augmenting $\mathrm{RBF}$, but rather restoring $\mathrm{RBF}$ when it is persistently inadequate.

The second theme is based on the notion that increasing the work of an injured organ may worsen outcomes. As in the example of myocardial infarction and ARDS, organ support during injury is a key approach to improving outcomes. Consistent with this concept, early institution of RRT has the capacity to rest the injured organ by limiting the solute load and mitigating fluid overload. As an example, much of the progress in abrogating the progression of CKD is focused on treating presumed hyperfiltration using renin-angiotensinaldosterone system blockade to lower the GFR and prevent fibrosis, thereby improving organ survival. Similarly, we believe that the utilization of early RRT and avoidance of GFR augmentation via hyperemia during 
AKI may have the same effect as angiotensin-converting enzyme inhibitors have on patients with CKD to preserve long-term renal function.

The third theme is based on recognizing that thermal control, antibiotic dosing and maintenance of electrolyte and volume balance must be carried out carefully during RRT to avoid harm. This is one of the core lessons from the experience of ARDS therapy; limiting the harm of the device-patient interaction. In order to improve outcomes, these aspects of the care of the patient with AKI must be improved.

The fourth theme is based on the preclinical and epidemiology data that show AKI is associated with progression to CKD. We posit that interventions initiated at the time of AKI may have durable long-term effects, particularly on retarding the progression of incident CKD. The improvement long-term outcomes include, but are not limited to, improvement in survival, a decreased incidence of CKD and fewer cardiovascular events.

\section{Testing the hypothesis: trial design}

We suggest a conceptual trial design to test our hypothesis. The study population would consist of patients with RIFLE/Acute Kidney Injury Network stage 2 or greater AKI who also have evidence of an elevated AKI biomarker that predicts a high likelihood of persistence or progression of AKI. Patients would then be randomized to one of three arms: standard care, protocolized AKI management, and protocolized AKI management plus experimental agent.

The standard care arm would involve conservative management and monitoring for a need to begin RRT. If required (as assessed by routine clinical judgment), CRRT should be delivered at a dose of 20 to $25 \mathrm{~cm}^{3} / \mathrm{kg} /$ hour for patients on vasopressors. Patients not requiring vasopressors should receive thrice-weekly hemodialysis with weekly $\mathrm{Kt} / \mathrm{V} \geq 3.6$. Additional management should be as per clinical judgment.

The AKI protocolized management arm would consist of early initiation of RRT based on the criteria presented in Table 1, and then protocolized CRRT management would be as follows: CRRT delivered at a dose of 20 to $25 \mathrm{~cm}^{3} / \mathrm{kg} /$ hour for patients on vasopressors, or thriceweekly hemodialysis with weekly delivered $\mathrm{Kt} / \mathrm{V} \geq 3.6$ for patients not requiring vasopressors; maintenance of normothermia unless otherwise indicated; avoidance of hypophosphatemia; antibiotic dosing based on delivered RRT dose; and maintenance of volume and electrolyte balance.

Multiple candidate agents could be tested in the protocolized AKI management plus therapeutic agent arm of the study. The goal of the therapeutic agent would be to decrease mortality, enhance renal recovery and decrease the incidence of long-term loss of renal
Table 1. Entry criteria for early initiation of renal replacement therapy

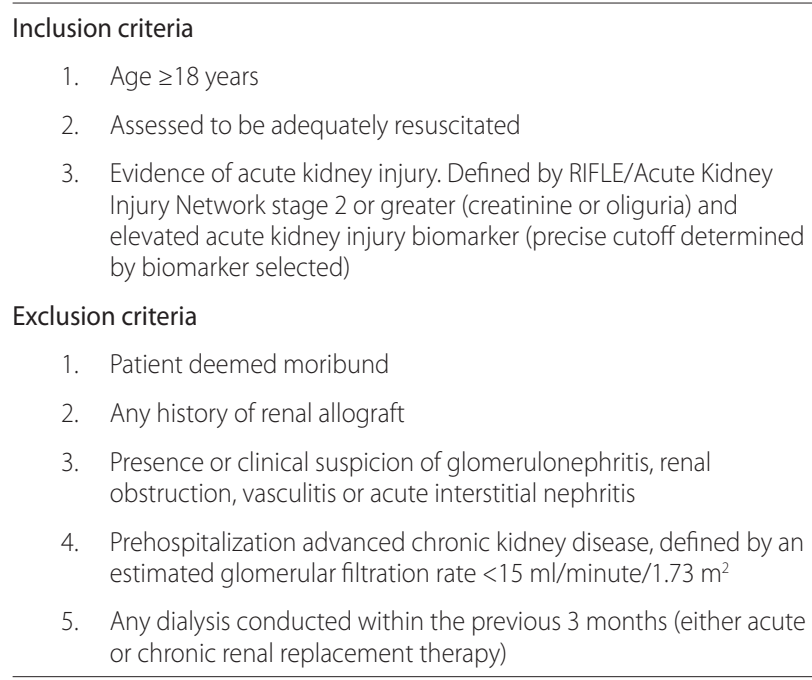

4. Prehospitalization advanced chronic kidney disease, defined by an estimated glomerular filtration rate $<15 \mathrm{ml} /$ minute $/ 1.73 \mathrm{~m}^{2}$

5. Any dialysis conducted within the previous 3 months (either acute or chronic renal replacement therapy)

function. As an example, preclinical models show that angiotensin-converting enzyme inhibition given at the time of AKI decreases the incidence of renal fibrosis during recovery [36]. Other agents that may prove successful include those that modulate the cell cycle or agents that block transforming growth factor beta or that target a variety of inflammatory pathways. Pilot and feasibility studies could start with variations of the approaches proposed.

\section{Conclusion}

AKI is a syndrome with a multitude of causes and is associated with a high risk for mortality or permanent loss of renal function. Our current understanding of the most common causes of AKI is limited. A change in the approach to AKI that shifts away from short-term endpoints such as death or dialysis and instead focuses on improving longer-term survival and mitigating permanent renal damage is likely to be more fruitful. In addition, we suggest that augmenting renal function via increasing the RBF or GFR during AKI may actually worsen outcomes. Resting the kidney and providing early RRT analogous to the approach in the treatment of ARDS may prove to be a more effective strategy to improve survival and decrease permanent loss of kidney function for patients with AKI.

\section{Abbreviations}

AKI, acute kidney injury; ARDS, adult respiratory distress syndrome; CKD chronic kidney disease; CRRT, continuous renal replacement therapy; GFR, glomerular filtration rate; RBF, renal blood flow; RIFLE, Risk, Injury, Failure, Loss and End-stage kidney disease; RRT, renal replacement therapy.

\section{Acknowledgements}

Aspects of this manuscript were presented and developed at the AKL Roundtable Discussion hosted by Jean-Louis Vincent in Brussels, Belgium in March 2011. The authors would like to thank Dr Vincent and all attendees of the roundtable. In addition, the authors would like to thank Patrick Brophy, 
Max Kökblach, Stuart Goldstein, Andrew Shaw, Patrick Murray, Sean Bagshaw Sheryl C Tilf, and Brucing Aurumboot for reviewing this manuscript.

\section{Competing interests}

All authors participated in the concept and writing of the manuscript. LSC has links with Gambro Medical, Nxstage Medical, Alere, Astute Medical and Abbott Medical. JAK has links with Gambro Medical, Alere Medical, Abbott, Fresenius, Baxter and Astute. CR has links with Gambro, Merck, Alere, Astute, Abbott and GE.

\section{Author details}

'Department of Anesthesiology and Critical Care Medicine, George Washington University Medical Center, 900 23 $3^{\text {rd }}$ Street, NW, RILF, Room G-105, Washington, DC 20037, USA. ${ }^{2}$ Department of Medicine, Division of Renal Diseases and Hypertension, George Washington University Medical Center, Washington DC 20037, USA. ${ }^{3}$ Department of Critical Care Medicine, University of Pittsburgh, Pittsburgh, PA, USA. ${ }^{4}$ CRISMA Center, Department of Critical Care Medicine, University of Pittsburgh School of Medicine, 3550 Terrace Street, 603B Scaife Hall Pittsburgh, PA 15261, USA. 'Department of Nephrology, Dialysis and Transplantation, International Renal Research Institute of Vicenza, San Bortolo Hospital, Viale Rodolfi, 37, 36100 Vicenza, Italy.

Published: 26 July 2012

\section{References}

1. Uchino S, Kellum JA, Bellomo R, Doig GS, Morimatsu H, Morgera S, Schetz M, Tan I, Bouman C, Macedo E, Gibney N, Tolwani A, Ronco C; Beginning and Ending Supportive Therapy for the Kidney (BEST Kidney) Investigators: Acute renal failure in critically ill patients: a multinational, multicenter study. JAMA 2005, 294:813-818.

2. Chertow GM, Burdick E, Honour M, Bonventre JV, Bates DW: Acute kidney injury, mortality, length of stay, and costs in hospitalized patients. J Am SoC Nephrol 2005, 16:3365-3370.

3. Jo SK, Rosner MH, Okusa MD: Pharmacologic treatment of acute kidney injury: why drugs haven't worked and what is on the horizon. Clin J Am SoC Nephrol 2007, 2:356-365

4. Ali T, Khan I, Simpson W, Prescott G, Townend J, Smith W, Macleod A: Incidence and outcomes in acute kidney injury: a comprehensive population-based study. J Am Soc Nephrol 2007, 18:1292-1298.

5. Langenberg C, Bagshaw SM, May CN, Bellomo R: The histopathology of septic acute kidney injury: a systematic review. Crit Care 2008, 12:R38.

6. Kellum JA: Impaired renal blood flow and the 'spicy food' hypothesis of acute kidney injury. Crit Care Med 2011, 39:901-903.

7. Hotchkiss RS, Swanson PE, Freeman BD, Tinsley KW, Cobb JP, Matuschak GM Buchman TG, Karl IE: Apoptotic cell death in patients with sepsis, shock, and multiple organ dysfunction. Crit Care Med 1999, 27:1230-1251.

8. Bahekar A, Singh M, Singh S, Bhuriya R, Ahmad K, Khosla S, Arora R: Cardiovascular outcomes using intra-aortic balloon pump in high-risk acute myocardial infarction with or without cardiogenic shock: a metaanalysis. J Cardiovasc Pharmacol Ther 2012, 17:44-56.

9. Liu KD, Matthay MA: Advances in critical care for the nephrologist: acute lung injury/ARDS. Clin J Am Soc Nephrol 2008, 3:578-586.

10. Van der Schueren G, Diltoer M, Laureys M, Huyghens L: Intermittent hemodialysis in critically ill patients with multiple organ dysfunction syndrome is associated with intestinal intramucosal acidosis. Intensive Care Med 1996, 22:747-751.

11. Davenport A: Dialytic treatment for septic patients with acute kidney injury. Kidney Blood Press Res 2011, 34:218-224.

12. Clive DM, Cohen AJ: Acute renal failure. In Irwin and Rippe's Intensive Care Medicine. 3rd edition. Philadelphia, PA: Lippincott Williams and Wilkins; 2003:889-990

13. Bouchard J, Soroko SB, Chertow GM, Himmelfarb J, Ikizler TA, Paganini EP, Mehta RL: Fluid accumulation, survival and recovery of kidney function in critically ill patients with acute kidney injury. Kidney Int 2009, 76:422-427.

14. Flori HR, Church G, Liu KD, Gildengorin G, Matthay MA: Positive fluid balance is associated with higher mortality and prolonged mechanical ventilation in pediatric patients with acute lung injury. Crit Care Res Pract 2011, 2011:854142.

15. Grams ME, Estrella MM, Coresh J, Brower RG, Liu KD: Fluid balance, diuretic use, and mortality in acute kidney injury. Clin J Am Soc Nephrol 2011, 6:966-973.

16. Wiedemann HP, Wheeler AP, Bernard GR, Thompson BT, Hayden D, deBoisblanc B, Connors AF, Jr, Hite RD, Harabin AL: Comparison of two fluidmanagement strategies in acute lung injury. N Engl J Med 2006 354:2564-2575.
17. Sutherland SM, Zappitelli M, Alexander SR, Chua AN, Brophy PD, Bunchman TE, Hackbarth R, Somers MJ, Baum M, Symons JM, Flores FX, Benfield M, Askenazi D, Chand D, Fortenberry JD, Mahan JD, McBryde K, Blowey D, Goldstein SL: Fluid overload and mortality in children receiving continuous renal replacement therapy: the prospective pediatric continuous renal replacement therapy registry. Am J Kidney Dis 2010, 55:316-325.

18. Seyler L, Cotton F, Taccone FS, De Backer D, Macours P, Vincent JL, Jacobs F: Recommended beta-lactam regimens are inadequate in septic patients treated with continuous renal replacement therapy. Crit Care 2011, 15:R137.

19. Zubert S, Funk DJ, Kumar A: Antibiotics in sepsis and septic shock: like everything else in life, timing is everything. Crit Care Med 2010, 38:1211-1212

20. Iregui M, Ward S, Sherman G, Fraser VJ, Kollef MH: Clinical importance of delays in the initiation of appropriate antibiotic treatment for ventilatorassociated pneumonia. Chest 2002, 122:262-268.

21. Palevsky PM, Zhang JH, O'Connor TZ, Chertow GM, Crowley ST, Choudhury D, Finkel K, Kellum JA, Paganini E, Schein RM, Smith MW, Swanson KM, Thompson BT, Vijayan A, Watnick S, Star RA, Peduzzi P: Intensity of renal support in critically ill patients with acute kidney injury. N Engl J Med 2008, 359:7-20.

22. Craddock PR, Yawata Y, VanSanten L, Gilberstadt S, Silvis S, Jacob HS: Acquired phagocyte dysfunction. A complication of the hypophosphatemia of parenteral hyperalimentation. N Engl J Med 1974, 290:1403-1407.

23. Amanzadeh J, Reilly RF, Jr: Hypophosphatemia: an evidence-based approach to its clinical consequences and management. Nat Clin Pract Nephrol 2006, 2:136-148.

24. Yagi N, Leblanc M, Sakai K, Wright EJ, Paganini EP: Cooling effect of continuous renal replacement therapy in critically ill patients. Am J Kidney Dis 1998, 32:1023-1030.

25. Laupland KB, Zahar JR, Adrie C, Schwebel C, Goldgran-Toledano D, Azoulay E, Garrouste-Orgeas M, Cohen Y, Jamali S, Souweine B, Darmon M, Timsit JF: Determinants of temperature abnormalities and influence on outcome of critical illness. Crit Care Med 2012, 40:145-151

26. Bellomo R, Cass A, Cole L, Finfer S, Gallagher M, Lo S, McArthur C, McGuinness S, Myburgh J, Norton R, Scheinkestel C, Su S: Intensity of continuous renalreplacement therapy in critically ill patients. N Engl J Med 2009, 361:1627-1638.

27. Liano F, Felipe C, Tenorio MT, Rivera M, Abraira V, Saez-de-Urturi JM, Ocana J, Fuentes C, Severiano S: Long-term outcome of acute tubular necrosis: a contribution to its natural history. Kidney Int 2007, 71:679-686.

28. Amdur RL, Chawla LS, Amodeo S, Kimmel PL, Palant CE: Outcomes following diagnosis of acute renal failure in U.S. veterans: focus on acute tubular necrosis. Kidney Int 2009, 76:1089-1097.

29. Chawla LS, Amdur RL, Amodeo S, Kimmel PL, Palant CE: The severity of acute kidney injury predicts progression to chronic kidney disease. Kidney Int 2011, 79:1361-1369.

30. Ishani A, Nelson D, Clothier B, Schult T, Nugent S, Greer N, Slinin Y, Ensrud KE: The magnitude of acute serum creatinine increase after cardiac surgery and the risk of chronic kidney disease, progression of kidney disease, and death. Arch Intern Med 2011, 171:226-233.

31. Lo LJ, Go AS, Chertow GM, McCulloch CE, Fan D, Ordonez JD, Hsu CY: Dialysis-requiring acute renal failure increases the risk of progressive chronic kidney disease. Kidney Int 2009, 76:893-899.

32. Wald R, Quinn RR, Luo J, Li P, Scales DC, Mamdani MM, Ray JG: Chronic dialysis and death among survivors of acute kidney injury requiring dialysis. JAMA 2009, 302:1179-1185.

33. Xue JL, Daniels F, Star RA, Kimmel PL, Eggers PW, Molitoris BA, Himmelfarb J, Collins AJ: Incidence and mortality of acute renal failure in Medicare beneficiaries, 1992 to 2001. J Am Soc Nephrol 2006, 17:1135-1142.

34. Lowe KG: The late prognosis in acute tubular necrosis; an interim followup report on 14 patients. Lancet 1952, 31:1086-1088.

35. Go AS, Chertow GM, Fan D, McCulloch CE, Hsu CY: Chronic kidney disease and the risks of death, cardiovascular events, and hospitalization. N Engl J Med 2004, 351:1296-1305.

36. Efrati S, Berman S, Hamad RA, Siman-Tov Y, Ilgiyaev E, Maslyakov I, Weissgarten J: Effect of captopril treatment on recuperation from ischemia/reperfusion-induced acute renal injury. Nephrol Dial Transplant 2012, 27:136-145.

doi:10.1186/cc11253

Cite this article as: Chawla LS, et al:: Permissive hypofiltration. Critical Care 2012, 16:317 